\title{
Myocardial Contrast Echocardiography has Favorable Sensitivity and Specificity for Coronary Artery Disease Diagnosis in Patients with LBBB: A Meta-analysis
}

\author{
Wissam Alajaji ${ }^{1}$, Atallah Baydoun ${ }^{2,3}$, Nathan Morris ${ }^{4}$, Leslie Henry ${ }^{5}$ and Brian D Hoit ${ }^{3,6 *}$ \\ ${ }^{1}$ Department of Internal Medicine, University Hospitals of Cleveland, Case Western Reserve University, Cleveland-Ohio, USA \\ ${ }^{2}$ Louis Stokes VA Medical Center, Cleveland-Ohio, USA \\ ${ }^{3}$ School of Medicine, Case Western Reserve University, Cleveland-Ohio, USA \\ ${ }^{4}$ Department of Epidemiology and Biostatistics, Case Western Reserve University, Cleveland-Ohio, USA \\ ${ }^{5}$ Core Library, University Hospitals of Cleveland, Case Western Reserve University, Cleveland-Ohio, USA \\ ${ }^{6}$ Division of Cardiovascular Medicine, Harrington Heart and Vascular Institute, Case Western Reserve University, Cleveland-Ohio, USA
}

\begin{abstract}
Background: Conflicting evidence exists on the ideal choice of non-invasive pharmacologic stress imaging for coronary artery disease (CAD) diagnosis in patients with left bundle branch block (LBBB). The aim of this metaanalysis is to compare data that examine the sensitivity and specificity of non-invasive pharmacologic stress imaging in patients with LBBB for obstructive CAD diagnosis.
\end{abstract}

Methods: We performed a literature search in MEDLINE, embase.com and Cochrane (CENTRAL) without publication type or language restrictions. Both pharmacologic stress echocardiography (SE) and nuclear myocardial perfusion imaging (MPI) searches were restricted to the period between January 2004 and review time. Exclusion criteria included studies that lacked sensitivity and specificity data. The primary objective was to compare the sensitivities and specificities of all pharmacologic SE, MPI, myocardial contrast echocardiography (MCE), stress cardiac magnetic resonance (CMR) and positron emission tomography (PET) for identifying significant CAD in patients with LBBB.

Results: 10 studies met the inclusion criteria for analysis. The sensitivity and specificity odds ratio of MCE was $92 \%$ (95\% Cl 81-97\%), 93\% (95\% Cl 86- 97\%); Dobutamine (D)-CMR 64\% (95\% Cl 42-82\%), 94\% (95\% Cl 85-98\%); pharmacologic SE 73\% (95\% Cl 55-86\%), 84\% (95\% Cl 75-91\%); and pharmacologic MPI 83\% (95\% Cl $72-91 \%), 56 \%(95 \% \mathrm{Cl} 42-70 \%)$.

Conclusion: MCE and D-CMR appear to have improved diagnostic accuracy in comparison to pharmacologic SE and MPI in patients with LBBB. Additional MCE and D-CMR studies are warranted given their potential to become the non-invasive gold standard for the diagnosis of CAD in this population.

Keywords: Non-invasive stress imaging; Left bundle branch block; Coronary artery disease diagnosis; Sensitivity; Specificity

\section{Introduction}

Pharmacologic stress imaging of the heart is considered to be the non-invasive test of choice for the diagnosis of obstructive coronary artery disease (CAD) in patients with left bundle branch block (LBBB) because of the non-specificity of exercise stress testing [1]. Pharmacologic stress echocardiography (SE) and nuclear myocardial perfusion imaging (MPI) are both widely available and less expensive than other forms of testing such as myocardial contrast echocardiography (MCE), positron emission tomography (PET), and cardiac magnetic resonance imaging (CMR). The current guidelines recommend pharmacologic stress testing with either MPI or SE as Class IB tests for stable ischemic heart disease evaluation in patients with LBBB, regardless of the ability to exercise [2]. Several studies attest to the diagnostic utility of both SE and MPI but the false positive rate remains higher in patients with than without LBBB [3,4]. In 2006, a meta-analysis pooling both exercise and pharmacologic data of stress imaging concluded that the MPI had higher overall sensitivity than SE ( $88.5 \%$ vs. $74.6 \%$ respectively, $\mathrm{p}<0.0001$ ) but had significantly lower specificity $(41.2 \%$ vs. $88.7 \%$ respectively, $\mathrm{p}<0.0001)$ for CAD in patients with LBBB [5]. More recent studies evaluated several interpretation strategies of stress imaging tests in patients with LBBB and reported less false positive rates in some of the studies than previously described.
For example, incorporating diastolic parameters during dobutamine SE increased the specificity for CAD diagnosis, and accounting for the presence of fixed LV cavity dilatation and LAD territory ischemia evaluation improved the diagnostic power of MPI in patients with suspected CAD and LBBB at baseline [6,7]. Moreover, technological advances such as MCE, stress CMR and PET imaging widened the options of non-invasive evaluation in this selected population with promising results [8]. Accordingly, we conducted this meta-analysis in order to compare the sensitivity and specificity of pharmacologic SE and MPI for obstructive CAD diagnosis over the past 10 years. In addition, we performed a literature search for studies that examined the sensitivity and specificity of the newer modalities, MCE, stress CMR, and PET for CAD in patients with LBBB.

*Corresponding author: Brian D Hoit, Division of Cardiovascular Medicine Harrington Heart and Vascular Institute, Case Western Reserve University Cleveland, USA, Tel: +216-844-3855; E-mail: bdh6@cwru.edu

Received April 14, 2016; Accepted April 28, 2016; Published May 09, 2016

Citation: Alajaji W, Baydoun A, Morris N, Henry L, Hoit BD (2016) Myocardial Contrast Echocardiography has Favorable Sensitivity and Specificity for Coronary Artery Disease Diagnosis in Patients with LBBB: A Meta-analysis. J Cardiovasc Dis Diagn S1: 003. doi:10.4172/2329-9517.S1-003

Copyright: (C) 2016 Alajaji W, et al. This is an open-access article distributed under the terms of the Creative Commons Attribution License, which permits unrestricted use, distribution, and reproduction in any medium, provided the original author and source are credited. 
Citation: Alajaji W, Baydoun A, Morris N, Henry L, Hoit BD (2016) Myocardial Contrast Echocardiography has Favorable Sensitivity and Specificity for Coronary Artery Disease Diagnosis in Patients with LBBB: A Meta-analysis. J Cardiovasc Dis Diagn S1: 003. doi:10.4172/2329-9517. S1-003

Page 2 of 8

Methods we published the research protocol of this systematic review online on PROSPERO systematic review database prior to data extraction and analysis on Feb-6-2015. The PROSPERO registration number is CRD42015016495.

The reference URLs for the published protocols are: http://www.crd. york.ac.uk/PROSPERO/display_record.asp?ID=CRD42015016495.

\section{Eligibility}

Inclusion criteria: We included studies that evaluated the sensitivity and specificity of pharmacologic SE, MPI, D-CMR, MCE, and PET for CAD diagnosis in patients with pre-existing LBBB. Exclusion criteria were case reports; reviews; studies that included patients with pacemaker or exercise induced LBBB, valvular heart disease, or that used exercise as a form of stress; and studies that did not compare non-invasive scan results to coronary angiography. The primary outcomes were comparisons of the sensitivities and specificities of DSE, adenosine MPI, MCE, and PET for identifying obstructive CAD ( $\geq 50 \%)$ in patients with LBBB.

Literature search: A literature search in MEDLINE, Embase. com, and Cochrane (CENTRAL) was conducted on February 22014. Language or publication type filters were not applied to the search strategy. We restricted the SE and MPI search to the period from 2004 until the current time of this review. The search was done under the supervision of the librarian (LH) and reviewed by the biostatistician (NM) and the co-authors; the search strategy was revised multiple times until the best strategy was achieved. A balanced combination of MeSH terms and keywords utilizing the various search options of the searched databases as shown in Appendix 1 was used. Ongoing research trials about the subject were searched in http://www.controlled-trials.com/ isrctn/ and http://www.clinicaltrials.gov. The detailed search strategy is summarized in Appendix 1. The search strategy was developed in compliance with the guidelines of peer review of electronic searches described by Sampson et al. [9].

Selection process two reviewers (WA, AB) screened the titles and abstracts of identified citations independently and in duplicate. The full texts for citations that were judged by at least one of the two reviewers as potentially eligible were subsequently retrieved and screened independently. The results of the screening process were compared, and any disagreement was resolved by either discussion or with the help of a third reviewer. Calibration exercises were conducted and standardized screening forms based on the eligibility criteria were used.

Data extraction Data were abstracted from eligible studies using standardized forms in duplication. Data collected included the type of study design, characteristics of the population, control, and outcomes.

Study characteristics: For each study, the noted characteristics included the year of publication, number of enrolled individuals, mean or frequency of male gender, age, cardiac risk factors, type of pharmacologic agent, the method of test interpretation used, QRS duration, and significant CAD definition. The non-invasive test was defined as positive for Ischemia when nuclear scans had any perfusion defect or wall motion abnormality; SE had any stress related regional wall motion abnormality (RWMA) or abnormal diastolic parameters; MCE had myocardial contrast opacification defect or impaired flow; or if CMR had any of the following: stress induced RWMA on cine exam, perfusion abnormality, or late gadolinium enhancement (LGE). Significant CAD definition was accepted if there was $\geq 50 \%$ stenosis in any major coronary artery by coronary angiography.
Quantitative data synthesis: The weighted mean frequency or mean for each of the mean age, male gender percent, angiographic CAD, hypertension, diabetes, LBBB definition, and QRS duration were calculated.

From each report, a $2 \times 2$ table was constructed summarizing the number of true/false positive results and true/false negative test results. The sensitivity and specificity were calculated from the $2 \times 2$ frequency data for pharmacologic MPI, SE, CMR, and MCE. Calculated values included the diagnostic sensitivity/specificity for each study along with the weighted (proportional to the sample size) mean sensitivity (number of true positives/number of patients with angiographically significant $\mathrm{CAD}$ ) and specificity (number of true negatives/total number of patients without angiographically significant CAD) from the combined data.

Risk of bias (quality) assessment: A risk of bias in each of the eligible studies was performed independently and in duplicate among two authors of this review using the Cochrane Risk of Bias tool. This tool includes the following criteria: lack of blinding of participants or study personnel; incompleteness of outcome data; selective outcome reporting, and other bias (Table 1). All studies were cross sectional and non-randomized. Two MPI and one MCE studies had unclear blinding of study personnel [10-12]. One had high risk for detection bias due to exclusion of patients who did not undergo coronary angiography within 6 month [7]; another excluded 6 patients from analysis due to suboptimal images [13]. One study had high risk attrition bias due to exclusion of basal segment data from analysis of all patients because of suboptimal images [10].

Otherwise, the remaining studies were considered low risk for bias for non-randomized data.

\section{Statistical analysis}

Methods: A meta-analysis of the diagnostic odds ratio (DOR) using the Der Simonian Laird approach was performed using Edgars test with funnel plots on the $\log (\mathrm{DOR})$ to assess publication bias [14]. Because there is typically a tradeoff between sensitivity and specificity, it is generally accepted that specialized methods are needed for the meta-analysis of diagnostic test data [15]. The bivariate approach $[15,16]$ as implemented in the mada package in $\mathrm{R}[17,18]$ which uses a multivariate normal prior was utilized to account for the correlation between sensitivity and specificity. The sensitivity of all included studies and the subgroup analysis of classes of the diagnostic tools were summarized.

Heterogeneity and Publication Bias Assessment There was evidence of study heterogeneity in the diagnostic odds ratio with a P-value of 0.007 for Cochran's Q Test of Heterogeneity and $\mathrm{I} 2=51 \%$. After accounting for the four diagnostic tools using meta-regression, the evidence for heterogeneity disappeared ( $\mathrm{P}$-value $=0.29, \mathrm{I} 2=14.91 \%)$. Thus other causes of heterogeneity were not investigated. There was not any convincing evidence of publication bias from Edgars test of funnel plot asymmetry $(\mathrm{P}$-value $=0.1234)$ or from visual inspection of the funnel plot (Figure 1).

\section{Results}

The search strategy identified 3013 citations ( 369 were duplicates and were removed). Of the 2644 titles, 2499 were non-relevant and excluded. Of the 145 abstracts, 135 were excluded (34 case reports, reviews of poster presentations and editorials, 32 prognosis, 20 excluded or lacked data on LBBB, 35 lacked sensitivity and specificity 
Citation: Alajaji W, Baydoun A, Morris N, Henry L, Hoit BD (2016) Myocardial Contrast Echocardiography has Favorable Sensitivity and Specificity for Coronary Artery Disease Diagnosis in Patients with LBBB: A Meta-analysis. J Cardiovasc Dis Diagn S1: 003. doi:10.4172/2329-9517. S1-003

Page 3 of 8

\begin{tabular}{|c|c|c|c|c|c|c|}
\hline \multirow[b]{2}{*}{ Study } & \multicolumn{2}{|c|}{ Selection Bias } & \multirow{2}{*}{$\begin{array}{l}\text { Performance Bias } \\
\text { Blinding of } \\
\text { Participant and } \\
\text { Personnel }\end{array}$} & \multicolumn{2}{|c|}{$\begin{array}{l}\text { Incomplete Outcome Reporting } \\
\text { (Attrition Bias) }\end{array}$} & \multirow{2}{*}{$\begin{array}{c}\text { Reporting Bias } \\
\begin{array}{c}\text { Selective Outcome } \\
\text { Reporting }\end{array}\end{array}$} \\
\hline & $\begin{array}{c}\text { Random Sequence } \\
\text { Generation }\end{array}$ & $\begin{array}{c}\text { Allocation } \\
\text { Concealment }\end{array}$ & & $\begin{array}{l}\text { Short Term } \\
\text { (2-6Weeks) }\end{array}$ & $\begin{array}{l}\text { Long Term (>6 } \\
\text { Weeks) }\end{array}$ & \\
\hline Badran et al. [6] & - & - & + & $?$ & $?$ & + \\
\hline Fal.lahi et al. [7] & - & - & + & $?$ & $?$ & + \\
\hline Hayat et al. [8] & - & - & + & $?$ & $?$ & + \\
\hline Karavidas et al. [10] & - & - & $?$ & $?$ & $?$ & - \\
\hline Feola et al. [11] & - & - & $?$ & $?$ & $?$ & + \\
\hline Felis et al. [12] & - & - & $?$ & $?$ & $?$ & + \\
\hline Soylu et al. [13] & - & - & + & $?$ & $?$ & - \\
\hline Pavlovic et al. [21] & - & - & $?$ & $?$ & $?$ & - \\
\hline Mordi et al. [22] & - & - & + & $?$ & $?$ & + \\
\hline Vigna et al. [27] & - & - & + & $?$ & $?$ & - \\
\hline
\end{tabular}

(-) High risk for bias; (?) Unclear risk for bias; (+) Low risk for bias.

Table 1: Cochrane tool for risk of bias scores of the various studies.

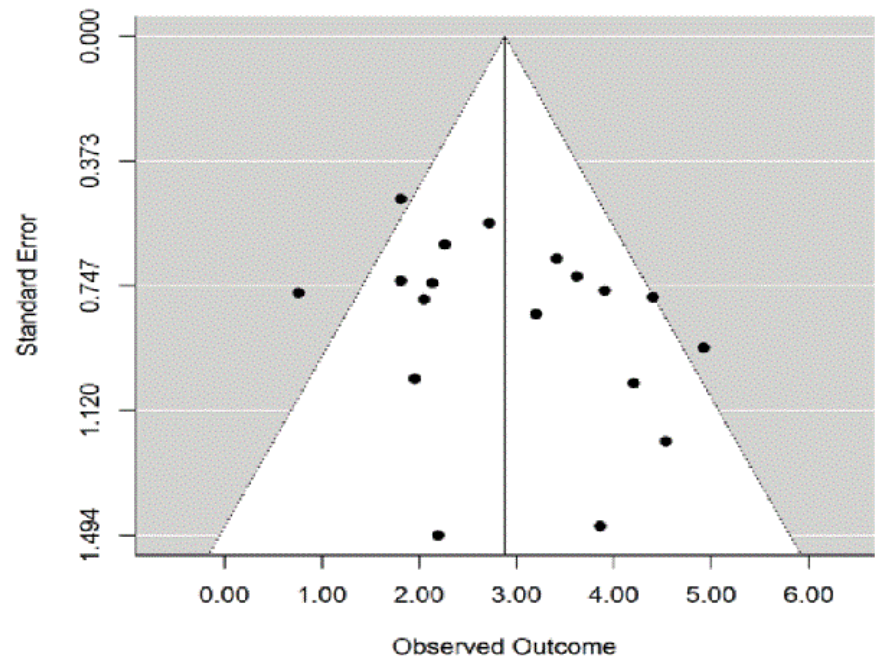

Figure 1: The funnel plot shows for each study the standard error vs. the observed diagnostic odds ratio. Some forms of publication bias would lead to asymmetry of the points around the central line.

data, 8 stress or pacing induced LBBB, 4 right bundle branch block (RBBB), 1 cardiac CT, 1 post MI) leaving 10 papers for analysis. A flow chart is attached in Appendix 1. The 10 eligible studies included: 3 SE $(\mathrm{n}=171), 7$ Nuclear MPI $(\mathrm{n}=326), 3$ MCE $(\mathrm{n}=125)$, and 1 dobutamine CMR ( $\mathrm{n}=82$ ) (Figure 2 and Table 2). Two SE studies addressed at the same time MPI and CMR, one for each. Two MCE studies also evaluated MPI sensitivity and specificity. All studies enrolled patients who were undergoing testing for suspected CAD with no prior history of myocardial infarction. The forest and crosshair plots [19]. Figures 3 and $4 \mathrm{~A}$ show the sensitivity and specificity of each study as well as the summarized sensitivity, specificity from the bivariate analysis. Characteristics of the study populations are summarized in Table 3.

\section{Sensitivity of non-invasive imaging for CAD detection in LBBB}

The overall sensitivity odds ratio of pharmacologic non-invasive stress imaging for CAD diagnosis in patients with LBBB was $77 \%$ (95\% CI, $68-84 \%)$. The overall highest odds ratio for sensitivity stratified by non-invasive test was for MCE 92\% (95\% CI, 81-97\%); this was followed by MPI with $83 \%$ (95\% CI, 72-91\%); pharmacologic SE with 73\% (95\%
CI, 55-86\%); and D-CMR with 64\% (95\% CI, 42-82\%) (Figure 3). PET scan had no data on sensitivity to answer our inquiry.

\section{Specificity of non-invasive imaging for CAD detection in LBBB}

The overall specificity odds ratio of pharmacologic non-invasive stress imaging for CAD diagnosis in patients with LBBB was $82 \%$ (95\% CI, 72-89\%). The overall specificities of individual non-invasive tools were highest for D-CMR and MCE with 94\% (95\% CI, 85-98\%) and 93\% (95\% CI, 86-97\%), respectively; this was followed by pharmacologic SE with 84\% (95\% CI, 75-91\%); and MPI with 56\% (95\% CI, 42-70\%) (Figure 3). PET scan had no data on specificity to answer our inquiry.

Summary of ROC curves, positive predictive and negative predictive values

The cross hair plot analysis of sensitivity and 1- specificity with the $95 \%$ confidence interval for each study demonstrates the relatively wider and lower range of specificities of nuclear MPI in comparison to the other modalities (Figure 4A). Analysis of the estimated sensitivity and 1- specificity and the associated underlying receiver operator curve 
Citation: Alajaji W, Baydoun A, Morris N, Henry L, Hoit BD (2016) Myocardial Contrast Echocardiography has Favorable Sensitivity and Specificity for Coronary Artery Disease Diagnosis in Patients with LBBB: A Meta-analysis. J Cardiovasc Dis Diagn S1: 003. doi:10.4172/2329-9517. S1-003

Number of Population Enrolled by The Different Studies

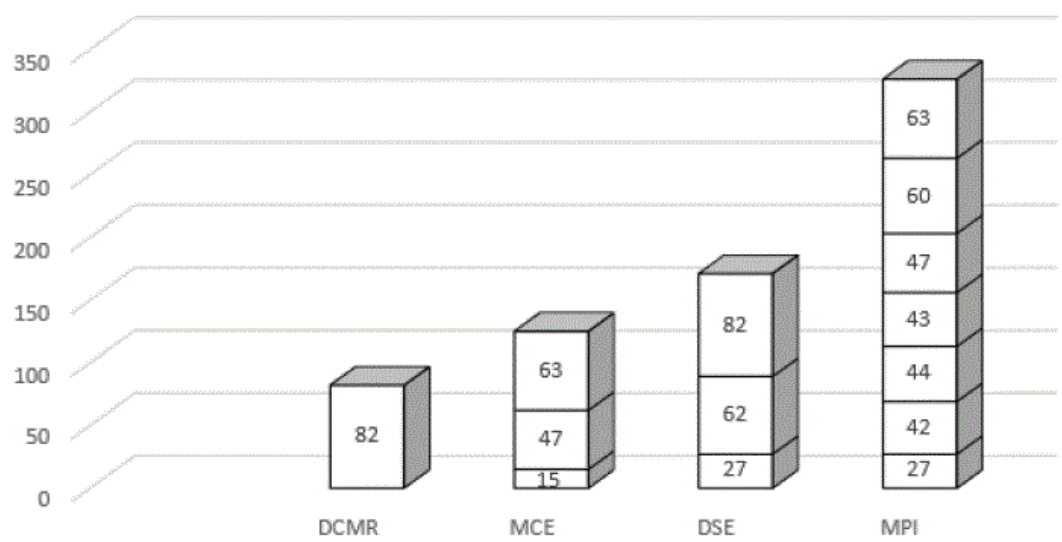

Figure 2: Stacked bar chart representing the sample size included in the individual studies stratified by non-invasive pharmacologic imaging tool.

\begin{tabular}{|c|c|c|c|c|}
\hline Study & Pharmacologic MPI & Pharmacologic SE & Pharmacologic CMR \\
\hline Badran et al. [6] & $(-)$ & 62 & $(-)$ \\
\hline Fal.lahi et al. [7] & 42 & $(-)$ & $(-)$ \\
\hline Hayat et al. [8] & 63 & $(-)$ & $(-)$ \\
\hline Karavidas et al. [10] & 47 & $(-)$ & $(-)$ \\
\hline Feola et al. [11] & 60 & $(-)$ & $(-)$ \\
\hline Felis et al. [12] & $(-)$ & $(-)$ & $(-)$ \\
\hline Soylu et al. [13] & 44 & $(-)$ & $(-)$ \\
\hline Pavlovic et al. [21] & 43 & $(-)$ & $(-)$ \\
\hline Mordi et al. [22] & $(-)$ & $(-)$ & \\
\hline Vigna et al. [27] & 27 & $(-)$ & $(-)$ \\
\hline
\end{tabular}

(-) No patients underwent this form of non-invasive testing; *Number of overlapping patients.

Table 2: Number of patients examined in each study with each non-invasive tool.

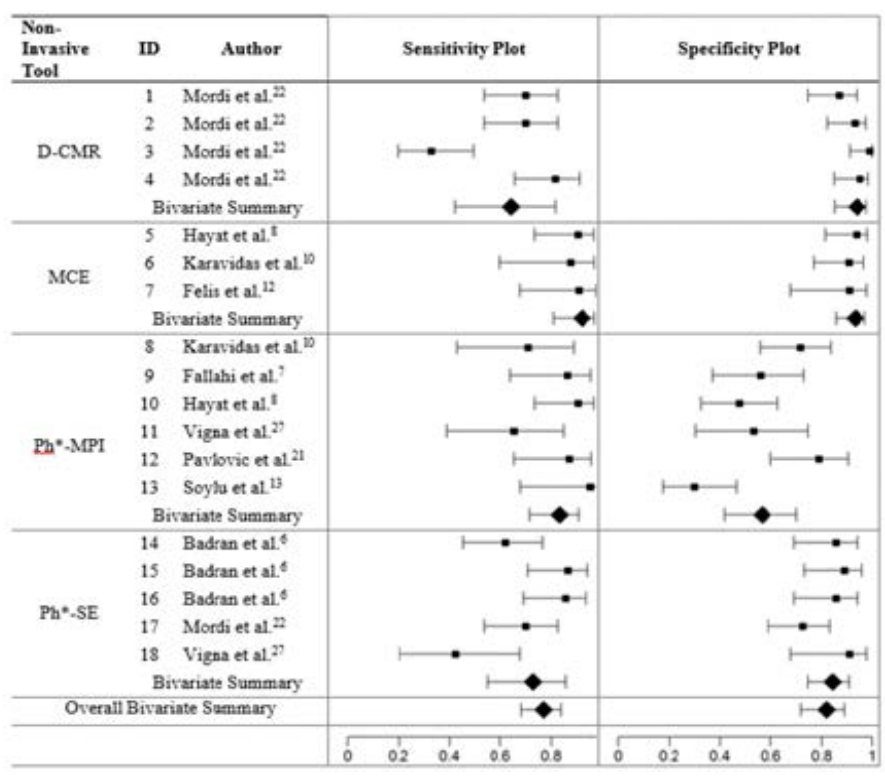

Repeated studies used the same population included in that study-1: Sensitivity and specificity by regional wall motion abnormality during D-CMR; 2 : Sensitivity and specificity of myocardial perfusion by D-CMR; 3: Sensitivity and specificity of late gadolinium enhancement by D-CMR; 4 : Sensitivity and specificity of comprehensive D-CMR (RWMA+perfusion+LGE); 14: Sensitivity and specificity of regional wall motion abnormality by echocardiography; 15: Sensitivity and specificity of peak systolic velocity of posterior septum, anterior septum, posterior, lateral, inferior, and anterior walls by tissue Doppler echocardiography; 16: Sensitivity and specificity of early diastolic velocity of posterior septum, anterior septum, posterior, lateral, inferior, and anterior walls tissue Doppler echocardiography.

Figure 3: Forest plots of sensitivities and specificities of the various studies stratified by the type on non-invasive stress imaging tools. *Ph: Pharmacologic. 
Citation: Alajaji W, Baydoun A, Morris N, Henry L, Hoit BD (2016) Myocardial Contrast Echocardiography has Favorable Sensitivity and Specificity for Coronary Artery Disease Diagnosis in Patients with LBBB: A Meta-analysis. J Cardiovasc Dis Diagn S1: 003. doi:10.4172/2329-9517. S1-003

Page 5 of 8
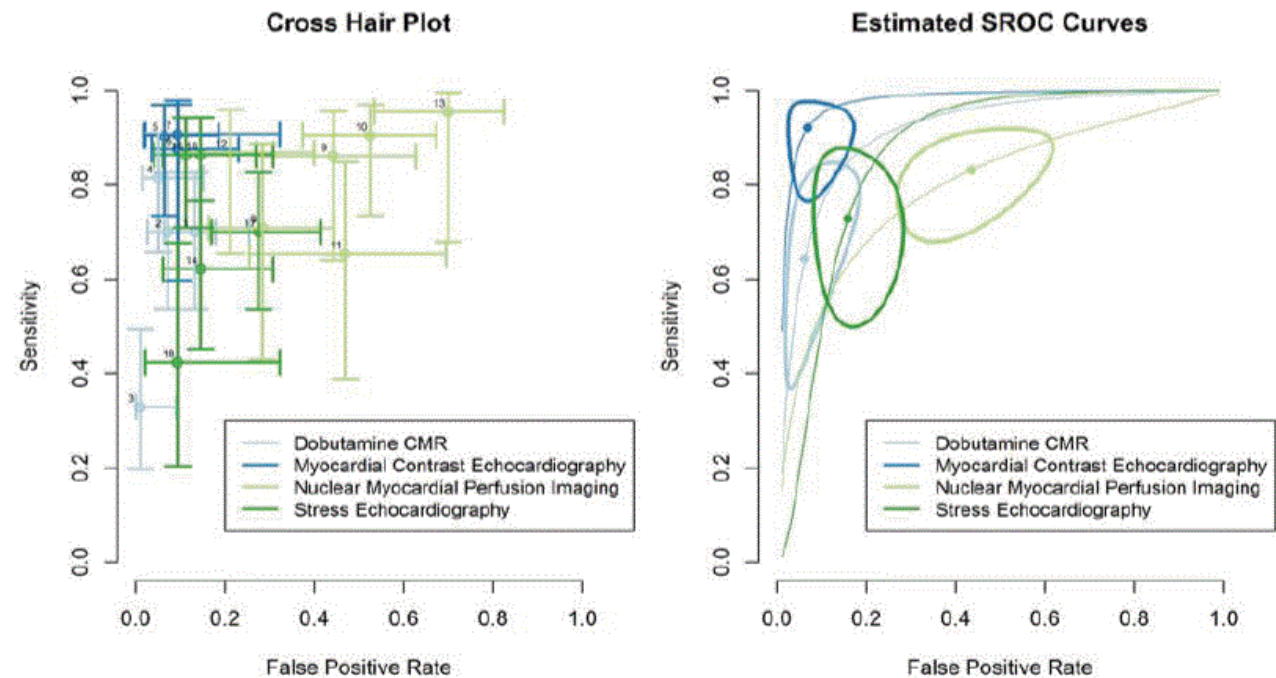

Figure 4: (Panel A) Jointly displays the 95\% confidence intervals for the sensitivity and 1-specificity for each study. Then numbers are the IDs from Figure 3. (Panel B) Displays the estimated sensitivity and 1-specificity of the subgroup and shows and estimated underlying receiver operator curve (SROC) for each subgroup.

\begin{tabular}{|c|c|c|c|c|}
\hline & Pharmacologic MPI & Pharmacologic SE & Pharmacologic CMR & MCE \\
\hline Year of Enrollment & $\begin{array}{l}\text { - } \quad \text { Aug/1998-Jun/2009 } \\
\text { - } N / A^{*}[7,8,13]\end{array}$ & - Jan/2002-Apr/2003 N/A* [6,22] & - N/A [22] & - Mar-Sep 2003 N/A* $[8,12]$ \\
\hline Total. Number (n) & -326 & - 171 & -82 & -125 \\
\hline Pharmacologic Agent & $\begin{array}{l}\text { - } \text { Adenosine }[10,11] \\
\text { - } \text { Dipyridamole }[7,8,13,21,27]\end{array}$ & $\begin{array}{l}\text { - } \text { Dobutamine }[6,22] \\
\text { - } \text { Dipyridamole [27] }\end{array}$ & - Dobutamine [22] & $\begin{array}{ll}\text { - } & \text { Adenosine [10] } \\
\text { - } & \text { Dipyridamole [8] } \\
\text { - } & \text { N/A }[12]\end{array}$ \\
\hline Indication & - CAD Diagnosis & - CAD Diagnosis & - CAD Diagnosis & - CAD Diagnosis \\
\hline Pretest Probability & $\begin{array}{ll}\text { - } & \text { Intermediate }[7,8,13,21] \\
- & \text { N/A }[10,11,27]\end{array}$ & $\begin{array}{ll}\text { - } & \text { Intermediate [22] } \\
\text { - } & \text { N/A }[6,27]\end{array}$ & - Intermediate [22] & $\begin{array}{ll}\text { - } & \text { Low [12] } \\
\text { - } & \text { Intermediate [8] } \\
\text { - } & \text { N/A [10] }\end{array}$ \\
\hline QRS Duration (ms) & $\begin{array}{ll}- & 129.6 \pm 26.4[11] \\
- & N / A^{*}[7,8,10,11,13,21]\end{array}$ & $\begin{array}{l}-133.0 \pm 8.1[22] \\
-\quad N / A^{*}[6,27]\end{array}$ & - $133.0 \pm 8.1[22]$ & - N/A* $[8,10,12]$ \\
\hline $\begin{array}{l}\text { Significant Stenosis } \\
\text { Definition }\end{array}$ & $\begin{aligned}- & \geq 50 \% \text { in any vessel } \\
& {[7,8,10,11,13,21] } \\
- & \geq 70 \% \text { in any vessel [27] }\end{aligned}$ & $\begin{aligned}- & \geq 50 \% \text { in any vessel [6] } \\
- & \geq 70 \% \text { in any vessel except } \\
& L M \geq 50 \%[22] \\
- & \geq 70 \% \text { in any vessel }[27]\end{aligned}$ & $\begin{aligned}- & \geq 70 \% \text { in any vessel except } \\
& L M \geq 50 \%[22]\end{aligned}$ & - $\quad \geq 50 \%$ in any vessel $[8,10,12]$ \\
\hline \multicolumn{5}{|l|}{ Clinical. Data } \\
\hline Mean Age \pm SD (years) & $-59.9 \pm 10.6$ & $-60.6 \pm 10.9$ & $-56.5 \pm 7.8$ & $-62.8 \pm 10.6$ \\
\hline$\%$ Mal.e & -27 & -41 & -53 & -30 \\
\hline LVEF $(\%)$ & $-50.3 \pm 13.6$ & $-49.3 \pm 6.7$ & $-N / A^{*}$ & $-47.5 \pm 16.0$ \\
\hline$\% \mathrm{HTN}$ & $-N / A^{*}$ & -36 & -38 & -22 \\
\hline$\%$ DM & $-N / A^{*}$ & -27 & -19 & -12 \\
\hline$\% \mathrm{DL}$ & $-N / A^{*}$ & -28 & -39 & -21 \\
\hline$\%$ Smoker & $-N / A^{*}$ & -33 & -33 & -13 \\
\hline
\end{tabular}

Weighted average characteristics of study populations (NA when more than hal.f the studies did not report, or no data was provided in an individual study).

Table 3: Characteristics of the studies and the enrolled population stratified by the non-invasive stress testing tool.

(SROC) for the four different non-invasive pharmacologic imaging tests revealed that the diagnostic accuracy of MCE was more favorable (Figure 4B). The C-index was highest for MCE with 0.97, followed by D-CMR with 0.91, pharmacologic SE with 0.868, and pharmacologic MPI with 0.795 . The positive predictive value is defined as the probability that an individual has CAD given that the test is positive, while a negative predictive value is the probability that an individual does not have CAD given that the test is negative. Both of these numbers may differ broadly depending on the prior probability that the individual has CAD (i.e., the prevalence of CAD in the individual's sub-population). Based on the point estimates of sensitivity and specificity, Figure 5 displays the positive and negative predictive values for a range of possible prior values.

\section{Discussion}

The meta-analysis described herein is the first to the best of our knowledge that compares the performance of contemporary pharmacologic stress tests for the initial CAD diagnosis in patients with pre-existing LBBB. It also serves as an update of a previous metaanalysis examining the diagnostic role of DSE and stress MPI in this same population [5]. 


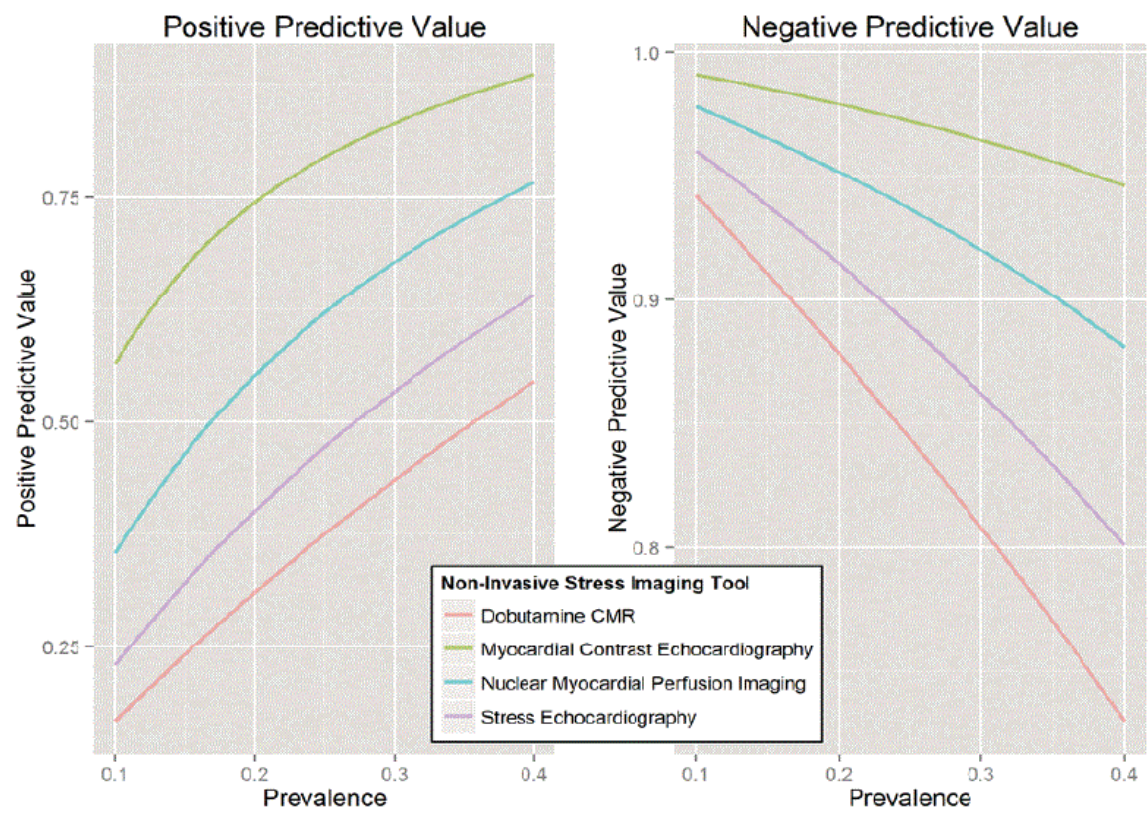

Figure 5: Both positive and negative predictive values may differ broadly depending on the prior probability that the individual has CAD (e.g. the prevalence of CAD in the individual's sub-population). These graphs display how (a) the positive predictive values and (b) the negative predictive values of the test change as a function of prevalence.

The main findings of this meta-analysis were 1) MCE has superior sensitivity and specificity characteristics compared to D-CMR, MPI and SE; 2) D-CMR and MCE have equally high specificity, but MCE is more sensitive; 3 ) Consistent with the older meta-analysis by Biagini et al. [5], MPI is more sensitive, but less specific than SE. These data suggest that $\mathrm{MCE}$ is a promising non-invasive imaging tool for CAD diagnosis in patients with LBBB. Also, the findings of this meta-analysis are supported by the findings of Hayat et al., Karavidas et al., Vigna et al., and Mordi et al. individual studies which compared more than one non-invasive tool.

\section{Non-invasive testing choice for obstructive CAD diagnosis in LBBB}

The non-invasive detection of obstructive CAD in patients with LBBB poses a diagnostic challenge. A careful choice of testing is warranted in order to avoid false positive results and unnecessary subsequent testing. Despite the current recommendation against exercise stress in general and ECG in particular for CAD diagnosis in this population, the ideal choice of non-invasive testing is not clear in the medical literature. At present, the pharmacologic MPI or SE is recommended in patients with LBBB. However, the detection of significant CAD by MPI is limited by the false positive rates of perfusion defects, particularly in the interventricular septum. Similarly, the diagnosis of RWMA involving the septum and apex during SE is frequently difficult in this population owing to pre-existing septal wall motion abnormalities. Non-invasive testing for CAD diagnosis can be achieved either by direct measurement of coronary flow utilizing perfusion scans (MPI, D-CMR or MCE) or indirectly by surrogates of coronary flow such as RWMA (CMR, SE) and diastolic function (SE). Unlike MPI, MCE and D-CMR offer real time assessment of flow. PET studies in LBBB reveal that asynchronous LV contractility leads to reduced septal vs. lateral wall contractility; as a result, in absence of flow limiting stenosis, counts appear more intense in lateral wall than septum. Similarly, low specificity characterizes MPI [20]. Based on the studies included in this meta-analysis, the performance of MPI seems to be highly affected by scan interpretation strategy. Soylu et al. reported that accounting for any reversible perfusion defect had high sensitivity $100 \%$ but at the expense of poor specificity $29 \%$, mostly due to defects in LAD distribution [13]. In addition, specificity was improved when scans were considered positive for defects with summed rest score (SRS) $\geq 12.4 \pm 5.5$ and summed difference score(SDS) $\geq 3.7 \pm 1.2$ with $89 \%$ sensitivity, and $80 \%$ specificity [21]. This observation warrants larger studies that compare the diagnostic accuracy of several MPI interpretation strategies in LBBB in order to make more accurate guideline recommendations as for how MPI scans need to be interpreted in LBBB.

In contrast, based on this meta-analysis, the real time assessment of coronary flow by CMR and MCE are more specific in LBBB. This may be related to the fact that these real time flow measurements are less subjected to the effect of LV asynchrony on flow than MPI or PET. First pass perfusion CMR has higher specificity (93.8\%) than cine CMR RWMA (87.5\%) with similar sensitivities of 70\% [22]. Gadolinium-enhanced CMR can assess myocardial opacification and washout durations in real time, which are markers of blood flow. The presence of late gadolinium enhancement carries a $100 \%$ specificity but at the sacrifice of a much lower sensitivity $(41.5 \%)$ in LBBB [22]. While surrogate ischemic markers by SE and CMR perform better that MPI, they do less favorably than methods that directly measure flow. The best overall performance in this meta-analysis was achieved with MCE. MCE perfusion imaging detects abnormalities that antedate RWMA by measuring the rate of echocardiographic contrast replenishment (reflecting myocardial red blood cell velocity; normal $<5$ seconds) following a destructive high energy ultrasound pulse. Replenishment takes longer when regional flow is reduced and is faster in hyperemic states [23]. Blood flow represents the product of velocity and plateau intensity, and when plateau intensity is normalized to LV cavity intensity, absolute myocardial blood flow can be calculated [23]. Indirect surrogates of ischemia detect CAD by uncovering RWMA or 
Citation: Alajaji W, Baydoun A, Morris N, Henry L, Hoit BD (2016) Myocardial Contrast Echocardiography has Favorable Sensitivity and Specificity for Coronary Artery Disease Diagnosis in Patients with LBBB: A Meta-analysis. J Cardiovasc Dis Diagn S1: 003. doi:10.4172/2329-9517. S1-003

Page 7 of 8

diastolic dysfunction. LV asynchronous contraction also complicates RWMA assessment and makes the indirect types of tests more subject to inter-observer variability in LBBB. Diastolic dysfunction in LBBB, particularly in the presence of LV hypertrophy, may be a poor surrogate of impaired coronary flow. Despite the fact that the diagnostic superiority of MCE similar to that revealed in this meta-analysis has not been demonstrated in large multicenter trials, the POINT BioMedical [24], RAMP-1 and 2 [25] did not address patients with LBBB. In a large multicenter study, Senior et al. reported superior sensitivity but lower specificity of MCE in comparison to SPECT [26]. However, this trial too did not include patients with LBBB [26]; the lower specificity was attributed to microvascular disease in the population studied [27]. Although the results of this meta-analysis are promising, further investigation in large multicenter trials is warranted to confirm the apparent diagnostic superiority of MCE in LBBB. Special population restrictions can limit the choice of testing. For example, CMR may not be applicable in patients with metal implants. Similarly, stress echo or MCE may not be an option for patients with poor acoustic windows. Future research examining the role of stepwise testing starting with MPI and proceeding to more specific non-invasive tools such as D-CMR or MCE prior to coronary angiography during the diagnostic algorithm for CAD in LBBB may be a reasonable approach. Limitations

Several potential limitations to this analysis should be acknowledged. First, the number of studies was quite small, especially for the subgroup analysis. This opens the possibility that estimates of study heterogeneity may be unreliable. Second, some of the studies used multiple ischemic markers and approaches; these studies were included in our analysis as independent studies. The small number of independents studies reflects a deficiency of the current literature. Third, several of the included studies suffered from multiple design limitations. Finally, the C-index summary measures as estimated by the bivariate analysis should not be over-interpreted as they involve extrapolation over areas of the ROC curve for which no data exist.

\section{Conclusion}

MCE and D-CMR appear to be promising tools in LBBB with superior diagnostic utility in comparison to DSE and nuclear MPI. Current datasets to guide informed decisions and guideline changes in LBBB are limited and additional studies using MCE and D-CMR in this population are warranted. CAD remains a diagnostic challenge in presence of $\mathrm{LBBB}$ and careful selection of non-invasive testing is necessary in order to avoid subsequent unnecessary testing given the high false positive rates in this population. Anatomic diagnosis remains the gold standard, and in selected cases, CAD diagnosis by coronary angiography may be necessary.

\section{References}

1. O'Keefe JH, Bateman TM, Barnhart CS (1993) Adenosine thallium-201 is superior to exercise thallium-201 for detecting coronary artery disease in patients with left bundle branch block. Journal of the American College of Cardiology 21: 1332-1338.

2. Fihn SD, Gardin JM, Abrams J, Berra K, Blankenship JC, et al. (2012) Guideline for the diagnosis and management of patients with stable ischemic heart disease: A report of the american college of cardiology foundation american heart association task force on practice guidelines, and the american college of physicians, american association for thoracic surgery, preventive cardiovascular nurses association, society for cardiovascular angiography and interventions, and society of thoracic surgeons. Journal of the American College of Cardiology 60: e44-e164

3. Demir H, Erbay G, Kir KM, Omurlu K, Berk F, et al. (2003) Clinical validation of technetium-99m mibi-gated single-photon emission computed tomography (spect) for avoiding false positive results in patients with left bundle-branch block: Comparison with stress-rest nongated spect. Clinical Cardiology 26 182-187.

4. Evangelista L, Nai Fovino L, Saladini F, Saladini G, Razzolini R, et al. (2012) Diagnostic and prognostic value of gated myocardial perfusion single-photon emission computed tomography in low-risk patients with left bundle-branch block. Nucl Med Commun 33: 491-497.

5. Biagini E, Shaw LJ, Poldermans D, Schinkel AF, Rizzello V, et al. (2006) Accuracy of non-invasive techniques for diagnosis of coronary artery disease and prediction of cardiac events in patients with left bundle branch block: A meta-analysis. Eur J Nucl Med Mol Imaging 33: 1442-1451.

6. Badran HB, Elnoamany MF, Seteha M (2007) Tissue velocity imaging with dobutamine stress echocardiography-a quantitative technique for identification of coronary artery disease in patients with left bundle branch block. Journal of the American Society of Echocardiography 20: 820-831.

7. Fallahi B, Beiki D, Eftekhari M, Gilani KA, Fard-Esfahani A, et al. (2009) High accuracy of myocardial perfusion imaging in patients with left bundle branch block: Comparison of four interpretation approaches. Hellenic Journal of Nuclear Medicine 12: 132-137.

8. Hayat SA, Dwivedi G, Jacobsen A, Lim TK, Kinsey C, et al. (2008) Effects of left bundle-branch block on cardiac structure, function, perfusion, and perfusion reserve: Implications for myocardial contrast echocardiography versus radionuclide perfusion imaging for the detection of coronary artery disease. Circulation 117: 1832-1841.

9. Sampson M, McGowan J, Cogo E, Grimshaw J, Moher D, et al. (2009) An evidence-based practice guideline for the peer review of electronic search strategies. Journal of Clinical Epidemiology 62: 944-952.

10. Karavidas Al, Matsakas EP, Lazaros GA, Brestas PS, Avramidis DA, et al. (2006) Comparison of myocardial contrast echocardiography with spect in the evaluation of coronary artery disease in asymptomatic patients with lbbb. International Journal of Cardiology 112: 334-340.

11. Feola M, Biggi A, Vado A, Ribichini F, Ferrero V, et al. (2004) The usefulness of adenosine 99mtctetrofosmin spect for the diagnosis of left anterior descending coronary artery disease in patients with chest pain and left bundle branch block. Nuclear medicine communications 25: 265-269.

12. Felis S, Deste W, Colonna P, Ragusa A, Scandura S, et al. (2005) Myocardia contrast echocardiography in the evaluation of myocardial perfusion in patients with left bundle branch block and coronary artery disease. Italian Heart Journal: Official Journal of the Italian Federation of Cardiology 6: 956-961.

13. Soylu O, Celik S, Karakus G, Yildirim A, Ergelen M, et al. (2008) Transthoracic doppler echocardiographic coronary flow imaging in identification of left anterior descending coronary artery stenosis in patients with left bundle branch block. Echocardiography 25: 1065-1070.

14. Viechtbauer W (2010) Conducting meta-analyses in $r$ with the metafor package. Journal of Statistical Software 36: 1-48.

15. Harbord RM, Deeks JJ, Egger M, Whiting P, Sterne JA (2007) A unification of models for meta-analysis of diagnostic accuracy studies. Biostatistics 8: 239251.

16. Reitsma JB, Glas AS, Rutjes AW, Scholten RJ, Bossuyt PM, et al. (2005) Bivariate analysis of sensitivity and specificity produces informative summary measures in diagnostic reviews. Journal of Clinical Epidemiology 58: 982-990.

17. Team RC (2015) R: A language and environment for statistical computing, $R$ Foundation for statistical Computing Vienna, Australia

18. Doebler P (2015) Mada: Meta-analysis of diagnostic accuracy.

19. Phillips B, Stewart LA, Sutton AJ (2010) 'Cross hairs' plots for diagnostic metaanalysis. Research Synthesis Methods 1: 308-315

20. Koepfli P, Wyss CA, Gaemperli O, Siegrist PT, Klainguti M, et al. (2009) Left bundle branch block causes relative but not absolute septal underperfusion during exercise. European Heart Journal 30: 2993-2999.

21. Pavlovic S, Sobic-Saranovic D, Djordjevic-Dikic A, Beleslin B, Stepanovic $\mathrm{J}$, et al. (2010) Comparative utility of gated myocardial perfusion imaging and transthoracic coronary flow reserve for the assessment of coronary artery disease in patients with left bundle branch block. Nuclear Medicine Communications 31: 334-340.

22. Mordi I, Stanton T, Carrick D, McClure J, Oldroyd K, et al. (2014) Comprehensive dobutamine stress $\mathrm{cmr}$ versus echocardiography in lbbb and suspected coronary artery disease. JACC: Cardiovascular Imaging 7: 490-498. 
Citation: Alajaji W, Baydoun A, Morris N, Henry L, Hoit BD (2016) Myocardial Contrast Echocardiography has Favorable Sensitivity and Specificity for Coronary Artery Disease Diagnosis in Patients with LBBB: A Meta-analysis. J Cardiovasc Dis Diagn S1: 003. doi:10.4172/2329-9517. S1-003

Page 8 of 8

23. Wei K, Jayaweera AR, Firoozan S, Linka A, Skyba DM, et al. (1998) Quantification of myocardial blood flow with ultrasound-induced destruction of microbubbles administered as a constant venous infusion. Circulation 97 473-483.

24. Wei K, Crouse L, Weiss J, Villanueva F, Schiller NB, et al. (2003) Comparison of usefulness of dipyridamole stress myocardial contrast echocardiography to technetium-99m sestamibi single- photon emission computed tomography for detection of coronary artery disease (pb127 multicenter phase 2 trial results). The American Journal of Cardiology 91: 1293-1298.

25. Senior R, Monaghan M, Main ML, Zamorano JL, Tiemann K, et al. (2009) Detection of coronary artery disease with perfusion stress echocardiography using a novel ultrasound imaging agent: Two phase 3 international trials in comparison with radionuclide perfusion imaging. European Heart Journal Cardiovascular Imaging 10: 26-35.

26. Senior R, Moreo A, Gaibazzi N, Agati L, Tiemann K, et al. (2013) Comparison of sulfur hexafluoride microbubble (sonovue)-enhanced myocardial contrast echocardiography with gated single-photon emission computed tomography for detection of significant coronary artery disease: A large european multicenter study. Journal of the American College of Cardiology 62: 1353-1361.

27. Vigna C, Stanislao M, De Rito V, Russo A, Santoro T, et al. (2006) Inaccuracy of dipyridamoleechocardiography or scintigraphy for the diagnosis of coronary artery disease in patients withboth left bundle branch block and left ventricular dysfunction. International Journal of Cardiology 110: 116-118. 


\section{Appendix1:}

A. Search strategy:

1 exp Bundle-Branch Block/ or exp heart conduction system/ or exp "bundle of his"/ or LBBB.mp. or (left* adj3 (bundl* or block*)).mp. or (conduction* adj3 disease*).mp. [mp=title, abstract, original title, name of substance word, subject heading word, keyword heading word, protocol supplementary concept word, rare disease supplementary concept word, unique identifier]

2 ((cardiac* or heart* or myocardi* or coronar* or ischemi* or ischaemi* or stress* or dobutamin* or adenosin* or dipyridamol* or vasodilat* or pharmacolog*) adj3 (nuclear* or perfusion* or SPECT* or imag* or echo*)).mp. [mp=title, abstract, original title, name of substance word, subject heading word, keyword heading word, protocol supplementary concept word, rare disease supplementary concept word, unique identifier]

3 exp echocardiography, stress/ or myocardial perfusion imaging/ or exp cardiac-gated imaging techniques/ or exp cardiac-gated single-photon emission computer-assisted tomography/

41 and (2 or 3$)$

5 limit 4 to $y r=" 2004$-Current"

6 ((cardiac* or heart* or myocardi* or coronar* or ischemi* or ischaemi* or stress* or dobutamin* or adenosin* or dipyridamol* or vasodilat* or pharmacolog*) adj3 (MRI or CMR or PET or ((contrast* adj3 echo*) or MCE))).mp. [mp=title, abstract, original title, name of substance word, subject heading word, keyword heading word, protocol supplementary concept word, rare disease supplementary concept word, unique identifier]

7 (cardiac* or heart* or myocardi* or coronar* or ischemi* or ischaemi* or stress* or dobutamin* or adenosin* or dipyridamol* or vasodilat* or pharmacolog*).mp. [mp=title, abstract, original title, name of substance word, subject heading word, keyword heading word, protocol supplementary concept word, rare disease supplementary concept word, unique identifier] 
8 exp magnetic resonance imaging/ or exp magnetic resonance imaging, cine/ or exp positronemission tomography/

96 or (7 and 8)

105 or 9

B. Flow Chart representing the number of citations retrieved by the search strategy and the number of the screened titles, abstracts and papers.

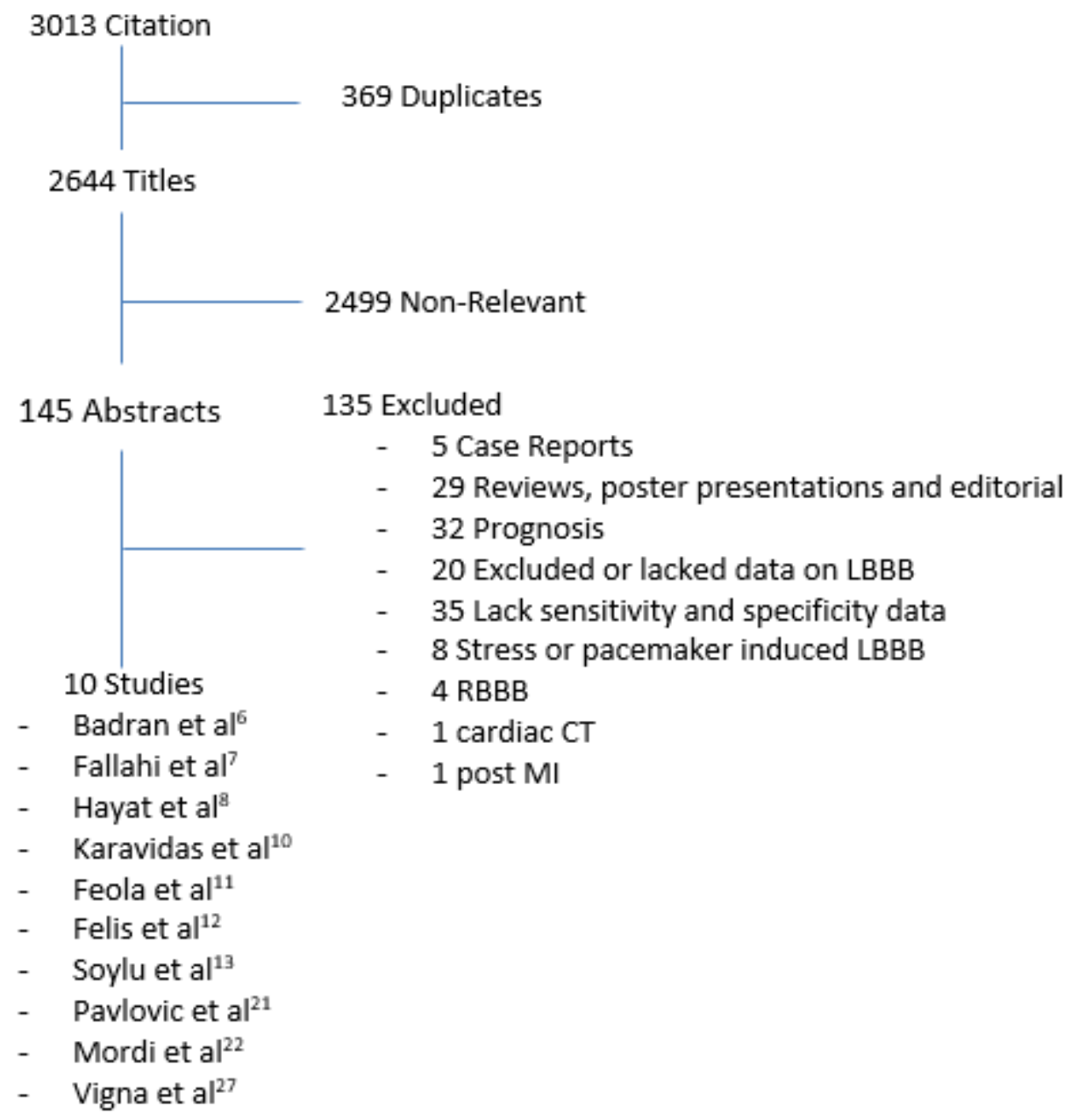

